\title{
Angle-resolved reflectivity of single-domain photonic crystals: Effects of disorder
}

\author{
Juan F. Galisteo Lòpez* and Willem L. Vos ${ }^{\dagger}$ \\ Van der Waals-Zeeman Instituut, ${ }^{\ddagger}$ Universiteit van Amsterdam, Valckenierstraat 65, 1018 XE Amsterdam, The Netherlands
}

(Received 12 March 2002; published 25 September 2002)

\begin{abstract}
Angle-resolved reflectivity has been measured from single-domain photonic crystals consisting of air spheres in a $\mathrm{TiO}_{2}$ backbone. Monochromatic beams are focused to a $10 \mu \mathrm{m}$ spot, and kept on the rotation axis with a precision better than $10 \mu \mathrm{m}$. We report high reflectivity up to $94 \%$ and surface domains as large as $200 \mu \mathrm{m}$, with a mosaic spread of $\pm 3^{\circ}$. The maximum reflectivity at normal incidence agrees well with theoretical calculations in the scalar-wave approximation, extended to include an extinction length due to diffuse scattering, that is obtained from independent experiments. The angle-resolved stop bands compare favorably with previous measurements using coarse beams as well as with prior theoretical calculations. Inhomogeneous broadening introduced by coarse beams in previous measurements is found to be small. Our results can only be understood if we assume multiple Bragg diffraction from more than one family of planes simultaneously to take place.
\end{abstract}

DOI: 10.1103/PhysRevE.66.036616

PACS number(s): 42.70.Qs, 42.25.Dd, 42.25.Fx, 81.05.Zx

\section{INTRODUCTION}

Following the proposition of the Purcell effect some decades ago [1], it has been suggested that a structure, called photonic crystal, can be tailored such that an excited atom placed inside would experience an inhibition of spontaneous emission [2]. A photonic crystal is a three-dimensional (3D) dielectric composite in which the refractive index is modulated with a periodicity of the order of the wavelength of light [2-4]. In such structures Bragg diffraction will forbid a certain range of frequencies to propagate for each direction of propagation. The extent of this forbidden range, known as stop gap, is dependent on the strength of interaction of light with the crystal, gauged by the polarizability per volume of the scatters that increases with the refractive index contrast [5]. The center frequency of the stop gap will depend on the lattice spacing, in qualitative accordance with Bragg's law [6]. For a sufficiently strong photonic interaction and suitable crystal structures, stop gaps can overlap for every direction of propagation leading to a range of frequencies for which light will not be allowed to propagate independent of direction, a photonic band gap. Driven by the important implications from the point of view of fundamental physics such as inhibition of spontaneous emission and localization of light [2-4], but also from the possibility of developing applications based on these structures [7-9], there is currently a large effort being carried out to fabricate the appropriate structure and also to understand the intricate ways in which light interacts with these structures.

When using white light to measure reflectivity from a photonic crystal, a stop gap will manifest as a peak in the

\footnotetext{
*Permanent address: Instituto de Ciencia de Materiales de Madrid (ICMM), Consejo Superior de Investigaciones Cientificas (CSIC), Cantoblanco 28049 (Madrid), Spain.

${ }^{\dagger}$ Permanent address: Faculty of Applied Physics and MESA+ Research Institute, University of Twente, PO Box 217, 7500 AE Enschede, The Netherlands; email address: w.l.vos@tn.utwente.nl

${ }^{\ddagger}$ URL: www.photonicbandgaps.com
}

reflected spectrum containing those frequencies that cannot propagate inside the crystal. These measurements will show a cross section of the electromagnetic dispersion relations of the crystal for a certain direction of propagation. On the other hand, if reflectivity is measured with monochromatic light as a function of angle of incidence, information will be obtained about the dispersion surfaces, that is, the sections at constant frequency of the dispersion relations. A complete characterization requires study of the dispersion relations for all frequencies and directions or wave vectors. For weakly interacting photonic crystals, reflectivity and transmission spectra are well described by the dynamical diffraction theory, which correctly describes photonic effects in x-ray diffraction $[10,11]$. In the absence of disorder, the width of the diffraction peak is equal to that of the stop gap, and measures the strength of interaction between crystal and incident light [5]. The height of the reflectivity peak will depend on the thickness of the crystal and the strength of the photonic interaction.

In the case of strongly photonic crystals, comparison with theory becomes more difficult. In strongly interacting photonic crystals, dynamical diffraction theory is not valid anymore, and it remains a challenge for theory to correctly describe reflectivity or transmission spectra from finite 3D crystals [2]. Another important challenge for theory is the fact that experimental spectra are inevitably affected by the presence of disorder. If coarse beams are used to measure reflectivity, it is likely that more than one domain will be probed, which can lead to inhomogeneous broadening of the reflectivity spectra $[12,13]$. If strain is present, each domain will contribute to the overall reflected spectrum with a peak centered at a different wavelength, whereas finite size domains contribute with a broadening [14], known as Scherrer broadening in $\mathrm{x}$-ray diffraction [10]. A mosaic spread will also hinder the interpretation of results. Grain boundaries, point defects, unstructured material and other defects in the bulk and on the surface of the samples, see e.g., Refs. $[16,17]$, will cause diffuse scattering, diminishing the reflected intensity, and complicating the test of new theories.

To minimize any spectral or angular features in the reflec- 


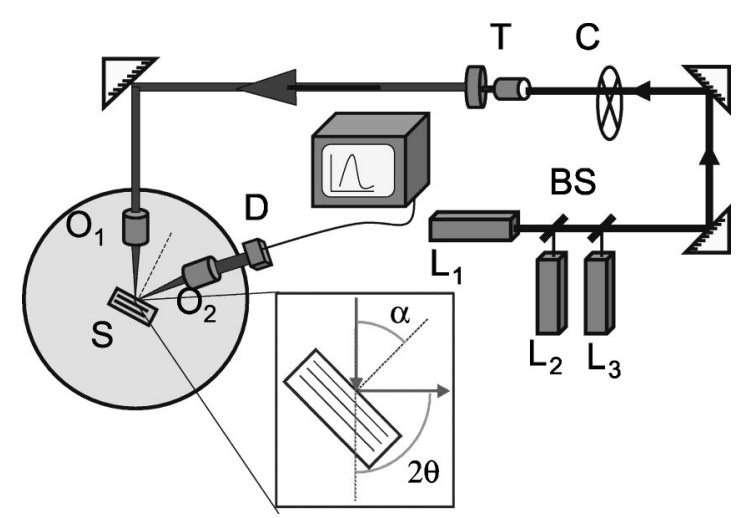

FIG. 1. Scheme of the experimental setup. Beams from lasers $L_{1,2,3}(633,850$, and $1064 \mathrm{~nm})$ are overlapped by beam splitters (BS). The resulting beam passes through a chopper (C) that is connected to a lock-in amplifier, and is expanded by a telescope (T). The microscope objective $\mathrm{O}_{1}$ focuses and $\mathrm{O}_{2}$ collects the diffracted light. In the inset, $\alpha$ and $2 \theta$ are defined as the angles that the incident beam forms with the normal to the surface and the diffracted beam, respectively.

tivity and transmission spectra due to inhomogenous broadening, it makes sense to try to probe single domains. This has been demonstrated at normal incidence using white light by Vlasov et al. $[13,15]$. In this paper, we probe the dispersion surface of photonic crystals that strongly interact with light, by measuring angle resolved reflectivity on single domains using monochromatic laser sources. In doing so, the effects of strain and mosaic spread are reduced, and the effects of mosaic spread and finite size are quantified. The effects of diffuse scattering are modeled by a small imaginary component of the dielectric function.

\section{EXPERIMENT}

The experimental setup is shown in Fig. 1. Three laser beams with frequencies of 633, 850, and $1064 \mathrm{~nm}$ are overlapped by a collection of beam splitters and modulated by a chopper. A telescope expands the beam which is then focused onto the sample. In order to combine a narrow focal spot of $10 \mu \mathrm{m}$ with a small angular aperture of $4^{\circ}$ full angle, we used microscope objectives with a focal length of 7.35 $\mathrm{cm}$ and a numerical aperture of 0.05 for both focusing and detection. The sample lies on the $\alpha$ rotation stage, where $\alpha$ is the angle between the incident beam and the normal to the surface of the sample (Fig. 1). The detection system consisting of a microscope objective and a diode detector is placed on a separate $2 \theta$ stage, with the same rotation axis as $\alpha ; 2 \theta$ is defined as the angle between the incident and the diffracted beams. Due to physical limitations imposed by the size of the objective holders, the angular range of collection is reduced to the interval $20^{\circ} \leqslant 2 \theta \leqslant 120^{\circ}$. An additional beam splitter is located before the focusing objective to allow for normal incidence collection at $2 \theta=180^{\circ}$.

The samples studied consist of a periodic $f c c$ array of air holes in a $\mathrm{TiO}_{2}$ matrix $[16,17]$. Several are the same as the ones in references $[12,18,19]$. We have employed samples of lattice parameters $847 \pm 5$ and $477 \pm 14 \mathrm{~nm}$ with several

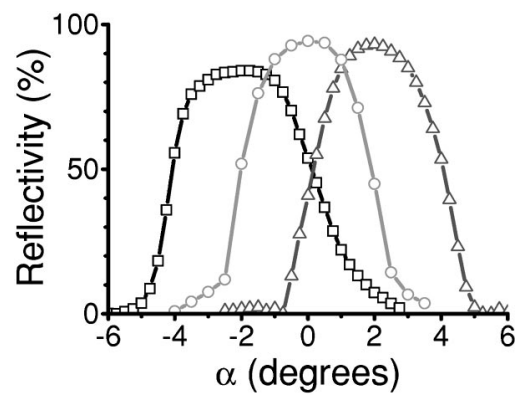

FIG. 2. Reflectivity for $a / \lambda=0.754 \pm 0.002(\mathrm{a}=477 \pm 14 \mathrm{~nm}$, $\lambda=633 \mathrm{~nm}$, sample $241-6$ ), measured as a function of $\alpha$ at different points on the sample surface. The $2 \theta$ detection stage remains fixed at normal incidence $\left(2 \theta=180^{\circ}\right)$.

wavelengths in order to measure reflectivity over a wide spectral range in terms of the relative frequency $a / \lambda$, where $a$ is the lattice parameter and $\lambda$ is the wavelength of light. For the samples and wavelengths used, we achieved values of $a / \lambda=0.754 \pm 0.002,0.796 \pm 0.005$, and $0.996 \pm 0.006$. For these values, we will probe inside the stop gap associated with the (111) Bragg diffraction, centered at $a / \lambda=0.73$ at normal incidence, as well as below and above the center of the avoided crossing of multiple Bragg diffraction at $a / \lambda$ $=0.85$ [18]. For $f c c$ photonic crystals band degeneracy can take place near the $U$ point, narrowing the stop gap for $p$-polarized light [20]. In order to avoid this, $s$-polarized light has been used in all our measurements.

\section{RESULTS}

We have measured reflectivity for different orientations $\alpha$ of the sample while maintaining the detector at a fixed position $2 \theta$ chosen so that the wavelength used is inside the stop gap for that orientation. By doing this we obtain information about the orientation of the different domains constituting the surface. Domains with different orientations will show reflectivity maxima centered at different sample orientations. We have measured reflectivity systematically in this way over the whole surface of the crystals, dividing the surface in a two dimensional grid consisting of points equidistant by $200 \mu \mathrm{m}$. In Fig. 2 normal incidence reflectivity results are shown for a sample with a lattice parameter $a=477 \mathrm{~nm}$ (sample 241-6) using a wavelength $\lambda=633 \mathrm{~nm}$, which for this sample is contained in the $L$ gap, that is, the stop gap for incidence normal to the (111) family of lattice planes. We observe three curves corresponding to measurements done on three different domains. Each curve shows an angular aperture equal to that of the reference measurement carried out with the mirror. Reflectivity as high as $94 \%$ has been found, confirming that good quality single crystals are being probed. From the position of the peaks, a mosaic spread of $\pm 2^{\circ}$ can be deduced. Similar measurements were carried out for a sample with a lattice parameter of $847 \mathrm{~nm}$ (sample 426-6) at a wavelength of $1064 \mathrm{~nm}$, only that $\alpha$ was increased to $30^{\circ}$ in order to be in the center of the stop gap. A reflectivity up to $77 \%$ and a mosaic spread of $\pm 3^{\circ}$ were recorded. In both sets of measurements, domains as large as $L=200 \mu \mathrm{m}$ were found, by selecting two points from the 


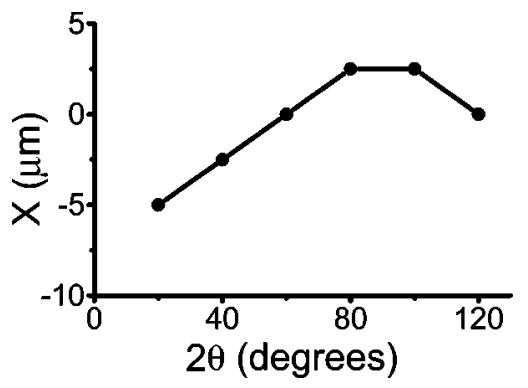

FIG. 3. Measured position of the rotation center of the $\alpha$ stage as a function of the angle rotated by the $2 \theta$ stage (circles, connected by lines). An overall change of less than $10 \mu \mathrm{m}$ is observed.

grid with the same orientation, repeating the measurements for intermediate points, and observing high reflectivities. The resulting Scherrer broadening as a result of finite size is estimated to be about $a /(\sqrt{3} L)=1 / 400$, which is only a small perturbation to the much broader widths of the photonic gaps [12].

In coarse beam experiments, the small mosaic spread observed would mean an inhomogeneous broadening of less than $15 \mathrm{~cm}^{-1}$ at normal incidence, increasing to a peak value of $344 \mathrm{~cm}^{-1}$ for an angle $\alpha=60^{\circ}$. These values of the broadening are small when compared with the widths of the peaks for those angles, $1004 \mathrm{~cm}^{-1}$ and $3795 \mathrm{~cm}^{-1}$, representing a relative broadening of only $1.5 \%$ and $8 \%$, respectively [12].

When trying to measure single domain angle resolved reflectivity, the beam must be carefully aligned with the rotation axis. Any misalignment will cause a translation of the region of the sample exposed to the beams, as well as bringing it out of focus as the sample is rotated. We found that the detection optics on the $2 \theta$ stage must be carefully counterweighted to avoid tilting of the rotation axis which also moves the sample out of focus. The counterweight process is done by observing the diffraction pattern of a $10 \mu \mathrm{m}$ thick tungsten wire located at the sample holder as the $2 \theta$ stage is rotated. The evolution of the pattern is observed for different positions of the counterweight. An alignment is achieved such that the wire moves less than $10 \mu \mathrm{m}$ from its original position over the whole angular range as is shown in Fig. 3. This result shows that it is feasible to carry out angle resolved scans on objects, including single crystals, of $10 \mu \mathrm{m}$ dimensions.

For the sample with $a=847 \mathrm{~nm}$, angle resolved reflectivity measurements were carried out for the frequencies $a / \lambda$ $=0.796$ and 0.996. The results are shown in Fig. 4. The excluded angular range is indicated as a crossed block. We see peaks that correspond to those directions where the stop gap contains the frequencies used. For $a / \lambda=0.796$ we observe a peak centered at $\alpha=30^{\circ}$ and showing a half width at half maximum of $13.2^{\circ}$, and a reflectivity of $77 \%$. The peak associated to $a / \lambda=0.996$ is centered at $\alpha=54^{\circ}$ and has a half width at half maximum of $10^{\circ}$ and up to $39 \%$ reflectivity. The shape of the peaks deviate from the flat-top predicted for perfect crystals by the dynamical diffraction theory. In this theory the rounded top shown by our peaks is associated with extinction, which in our case can only come from dif-

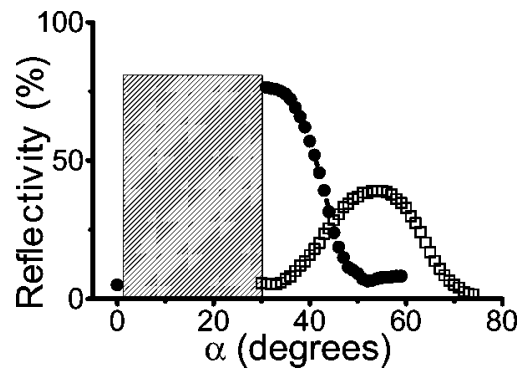

FIG. 4. Angle-resolved reflectivity on sample 426-6 ( $a$ $=847 \mathrm{~nm}$ ) for $a / \lambda=0.796$ (solid circles: $\lambda=1064 \mathrm{~nm}$ ) and $a / \lambda$ $=0.996$ (open squares: $\lambda=850 \mathrm{~nm}$ ) as a function of $\alpha$. The collection is done at $2 \theta=180-2 \alpha$ for every $\alpha$. The hatched rectangle indicates the excluded angular region due to physical limitations in the setup.

fuse scattering by defects, since for the range of wavelengths that we are using $\mathrm{TiO}_{2}$ does not absorb [17]. We can also see that as the reflectivity peak appears for larger angles, the reflected intensity decreases. This cannot come from any misalignment of the beam with respect to the rotation center, since in the reference measurements taken with the mirror, a signal constant within 5\% was recorded at all angles. It is conceivable that diffracted energy is carried away by multiple Bragg diffraction discussed below, which warrants further study.

To obtain the range where stop gaps are present, we have used the heuristic but reliable criterion [5] that the full width at half maximum of a reflectivity peak corresponds to a stop gap. Data obtained in this way are plotted in Fig. 5. The single point at normal incidence corresponds to the measurements done at relative frequency $a / \lambda=0.754$, see Fig. 3 . The hatched line at $a / \lambda=0.796$ is our estimation of the fraction of the stop gap falling within the forbidden angular range. At $a / \lambda=0.996$ two peaks centered at different angles were obtained in separate domains, probably due to strain. Mosaic spread is not likely to be the reason for these two peaks, since each domain was properly oriented with respect to the incident beam by measurements similar to those shown in Fig. 3. The peaks are plotted together with error bars indicating the uncertainty in the width.

The triangles in Fig. 5 indicate previous measurements with coarse beams $[12,18,19]$. For the relative frequency $a / \lambda=0.796$, a good agreement is observed between previous and present measurements. At $a / \lambda=0.996$, the results ob-

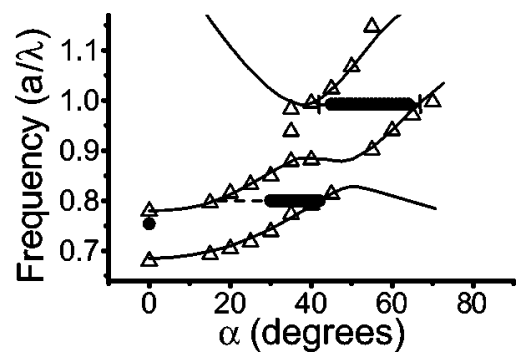

FIG. 5. Comparison of present angle-resolved measurements (horizontal lines) with previous measurements using coarse beams (open symbols) and theoretical predictions (drawn curves). 
tained with coarse beams show a broader stop gap than that obtained with a focussed beam. A reason for this deviation could be that for measurements with focussed beams, a realignment is carried out for each domain as described above, in order to avoid any inhomogeneous broadening due to mosaic spread. But for the case of coarse beams many domains are probed simultaneously, making it impossible to realign each of them and therefore introducing inhomogeneous broadening, which for this angular range can reach values up to $8 \%$ in frequency as discussed above.

The drawn curves in Fig. 5 are dispersion relations calculated with the plane wave expansion using a dielectric model described in Refs. [18] and [19]. For normal incidences this model reveals diffraction by only the (111) family of planes taking place. As the angle of incidence increases we move along the surface of the Brillouin zone in reciprocal space. As we reach the $U$ point, diffraction from the (111) and (200) families of planes will take place simultaneously resulting in band repulsion of Bloch states [18]. The fact that many waves couple, will cause a deviation of the dispersion relation from simple Bragg behavior, thereby flattening the photonic bands, a requirement for the formation of a photonic band gap. As shown in Fig. 5, the relative frequencies we have used in our experiments fall above and below the avoided crossing, and show good agreement with the calculated bands. Any attempt to explain similar results with a model based on simple Bragg diffraction will therefore lead to systematic errors.

\section{INTERPRETATION}

To theoretically interpret our results at normal incidence, we have performed calculations using the so-called scalarwave approximation, that is, using the plane-wave expansion in the $E$-field formulation and retaining only two reciprocal lattice vectors, similar to Refs. [21,22]. We have extended the model to include extinction as a result of scattering from crystal imperfections. The two reciprocal lattice vectors correspond to the incident wave $h k l=000$, and the diffracted wave $h k l=111$. The Fourier components of the dielectric function are derived from the model described in Refs. $[18,19]$, that accurately mimic the structure of our crystals, and whose dispersion relations agree well with reflectivity experiments. We take extinction due to diffuse scattering by for instance point defects and grain boundaries into account by a complex component of the average dielectric constant of the crystal $\epsilon^{\prime \prime}(000)$, which corresponds to a uniform extinction throughout the crystal. Note that this is different from the situation in x-ray diffraction, where absorption can only be present at the positions of atoms, and the extinction is thus spatially modulated $[10,11]$. Figure 6 (inset) shows a calculated reflectivity curve as a function of frequency. While we have not measured such a frequency dependence (see e.g., Refs. $[13,15]$ ), we can nevertheless compare the reflectivity measured at normal incidence to the maximum $R_{\max }$ of the calculated peak. The dashed curve in Fig. 6 shows $R_{\max }$ as a function of the number of lattice planes in the absence of extinction, that is, with a purely real $\epsilon^{\prime}(000)$. It is seen that $R_{\max }$ strongly increases, with a Bragg length of

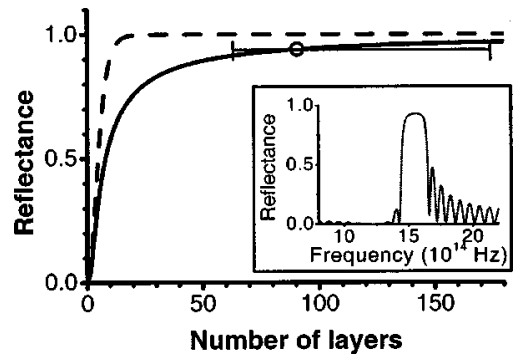

FIG. 6. The dotted line shows the maximum reflected intensity $R_{\max }$ as a function of the crystal thickness. The solid line represents the increase of $R_{\max }$ with the extinction length $l_{\text {ext }}$, for a 40-layerthick crystal. Error bars indicate the $l_{\text {ext }}$ range that accounts for the reflectivity we observe experimentally. Inset: calculated reflectivity spectrum at normal incidence for a crystal in the presence of extinction.

about seven layers where $R_{\max } \sim 1-(1 / e)$, before saturating at near unity for more than 20 planes. The solid curve in Fig. 6 shows $R_{\max }$ for a 40-plane crystal as a function of the extinction length $l_{\text {ext }}$, expressed in the number of layers. It is seen that the maximum reflectivity increases with extinction length. From the difference between $R_{\max }$ versus structure thickness in otherwise perfect crystals (dashed) and $R_{\max }$ versus $l_{\text {ext }}$ (drawn), it is obvious that reflectivity in presence of extinction cannot be simply regarded as reflectivity from a crystal with finite thickness. For an $l_{\text {ext }}$ between 65 and 170 planes, we obtain a maximum reflectivity of $94 \pm 3 \%$ as observed in the present experiment (Fig. 2). Such an extinction length compares favorably with mean free paths of 10 to $20 \mu \mathrm{m}$ or 60 layers, that were measured earlier by Koenderink et al. [23]. The extinction length corresponds to a small ratio between the imaginary and real components of the dielectric function of $\epsilon^{\prime \prime}(000) / \epsilon^{\prime}(000)=0.0035$. It is interesting to consider how long an extinction length is required to obtain a maximum reflectivity of $99.99 \%$, that is necessary for mirrors surrounding high- $Q$ cavities [24]. For a crystal with a thickness larger than 20 layers, the calculated $l_{\text {ext }}$ to obtain such a reflectivity is of the order of $10^{4}$ layers, which would require considerable improvements of all current fabrication techniques [5].

It is also interesting to note that a complex $\epsilon^{\prime \prime}(000)$ has been used for two-dimensional photonic crystal slabs by Benisty et al., to describe the results of out-of-plane losses [25]. In their case, a ratio $\epsilon^{\prime \prime}(000) / \epsilon^{\prime}(000)$ of 0.11 was found. In the light of the paucity of similar results, we conclude that extinction is a seriously underestimated issue in photonic crystal research, which requires much more attention prior to considering applications.

\section{CONCLUSIONS}

We have measured angle-resolved reflectivity with laser beams focussed to within single domains. Special care has been taken to assure an alignment of the waist of the beam with the axis of rotation to a precision of $10 \mu \mathrm{m}$. This technique proves valid to carry out reflectivity measurements on samples of $10 \mu \mathrm{m}$ in dimensions. We have observed high reflectivities up to $94 \%$ at normal incidence. This value de- 
creases for larger angles of incidence, a behavior not understood at this time. Evidence has been found for small inhomogeneous broadening present in previous measurements with coarse beams, due to the presence of a mosaic spread of $\pm 3^{\circ}$, and finite size effects are found to be less than about $1 / 400$. This technique opens an exciting opportunity to study light propagation in a photonic single crystal for any direction through the determination of the group velocity and the group velocity dispersion using pulsed laser beams, which has only been done for normal incidence [26,27]. With suitable tunable laser sources, this method proves ideal to fully characterize dispersions surfaces of any photonic crystal. These measurements have given us valuable in situ informa- tion about the structure of the surface of our crystals, which complements that obtained from optical and scanning electron microscopy.

\section{ACKNOWLEDGMENTS}

We would like to thank Femius Koenderink for experimental help and discussions and Ad Lagendijk for general support. This work was part of the research program of the "Stichting voor Fundamenteel Onderzoek der Materie" (FOM), which was supported by the "Nederlandse Organisatie voor Wetenschappelijk Onderzoek" (NWO).
[1] E.M. Purcell, Phys. Rev. 69, 2059 (1946).

[2] Photonic Crystals and Light Localization in the 21st Century, Vol. 563 of Nato Advanced Studies Institute, Series C: Mathematical and Physical Sciences, edited by C.M. Soukoulis (Kluwer, Dordrecht, 2001).

[3] J.D. Joannopoulos, R.D. Meade, and J.N. Winn, Photonic Crystals, Molding the Flow of Light (Princeton University Press, Princeton, 1995).

[4] A.F. Koenderink, P.M. Johnson, J.F. Galisteo Lòpez, and W.L. Vos, C. R. Phys. 3, 67 (2002).

[5] W.L. Vos, H.M. van Driel, M. Megens, A.F. Koenderink, and A. Imhof, in Photonic Crystals and Light Localization in the 21st Century (Ref. [2]), pp. 191-218.

[6] W.L. Vos, R. Sprik, A. van Blaaderen, A. Imhof, A. Lagendijk, and G.H, Wegdam, Phys. Rev. B 53, 16231 (1996); 55, 1903 (1997).

[7] H. Kosaka, T. Kawashima, A. Tomita, M. Notomi, T. Tamamura, T. Sato, and S. Kawakami, Phys. Rev. B 58, 10096 (1998).

[8] O. Painter, R.K. Lee, A. Scherer, A. Yariv, J.D. O'Brien, P.D. Dapkus, and I. Kim, Science 284, 1819 (1999).

[9] C.J.M. Smith, R.M. De La Rue, M. Rattier, S. Olivier, H. Benisty, C. Weisbuch, T.F. Krauss, R. Houdré, and U. Oesterle, Appl. Phys. Lett. 78, 1487 (2001).

[10] R.W. James, The Optical Principles of the Diffraction of $X$-Rays (Bell, London, 1954).

[11] W.H. Zachariasen, Theory of X-ray Diffraction in Crystals (Wiley, New York, 1945).

[12] M.S. Thijssen, R. Sprik, J.E.G.J. Wijnhoven, M. Megens, T.
Narayanan, A. Lagendijk, and W.L. Vos, Phys. Rev. Lett. 83, 2730 (1999).

[13] Y.A. Vlasov, M. Deutsch, and D.J. Norris, Appl. Phys. Lett. 76, 1627 (2000).

[14] J.F. Bertone, P. Jiang, K.S. Hwang, D.M. Mittleman, and V.L. Colvin, Phys. Rev. Lett. 83, 300 (1999).

[15] Y.A. Vlasov, X.Z. Bo, J.C. Sturm, and D.J. Norris, Nature (London) 414, 289 (2001).

[16] J.E.G.J. Wijnhoven and W.L. Vos, Science 281, 802 (1998).

[17] J.E.G.J. Wijnhoven, L. Bechger, and W.L. Vos, Chem. Mater. 13, 4486 (2001).

[18] H.M. van Driel and W.L. Vos, Phys. Rev. B 62, 9872 (2000).

[19] W.L. Vos and H.M. van Driel, Phys. Lett. A 272, 101 (2000).

[20] K.M. Ho, C.T. Chan, and C.M. Soukoulis, Phys. Rev. Lett. 65, 3152 (1990).

[21] K.W.K. Shung and Y.C. Tsai, Phys. Rev. B 48, 11265 (1993).

[22] D.M. Mittleman, J.F. Bertone, P. Jiang, K.S. Hwang, and V.L. Colvin, J. Chem. Phys. 111, 345 (2000).

[23] A.F. Koenderink, M. Megens, G. van Soest, W.L. Vos, and A. Lagendijk, Phys. Lett. A 268, 104 (2000).

[24] P. Münstermann, T. Fischer, P. Maunz, P.W.H. Pinkse, and G. Rempe, Phys. Rev. Lett. 82, 3791 (1999).

[25] H. Benisty, D. Labilloy, C. Weisbuch, C.J.M. Smith, T.F. Krauss, D. Cassagne, A. Beraud, and C. Jouanin, Appl. Phys. Lett. 76, 532 (2000).

[26] Y.A. Vlasov, S. Petit, G. Klein, B. Hönerlage, and C. Hirlimann, Phys. Rev. E 60, 1030 (1999).

[27] A. Imhof, W.L. Vos, R. Sprik, and A. Lagendijk, Phys. Rev. Lett. 83, 2942 (1999). 